\section{La cigale ou la fourmi, ou quoi faire de ses gains...}

La surprise $a$, semble-t-il, été divine: les comptes 2000 de la Confédération affichent un excédent de recettes de 4,5 milliards de francs là, où, le budget prévoyait pourtant un déficit de 1,8 milliards de francs. Ce sont donc 6,3 milliards de francs de plus qui sont tombés dans les caisses de notre Etat fédéral et la première question que se pose l'ensemble de la classe politique est: qu'en faire?

Le syndic de n'importe quelle petite commune $d u$ Gros de Vaud sait qu'il lui appartient de gérer ses fonds de façon à les investir en période de basse conjoncture et d'économiser, voire de diminuer la dette, en période de haute conjoncture au moment où les impôts rentrent de façon satisfaisante.

L'ensemble de la classe politique s'est donc félicitée de cette excellente nouvelle fédérale, tout en critiquant sévèrement l'absence de vues prospectives $d u$ Conseiller fédéral Kaspar Villiger pour les erreurs d'estimation enregistrées dans les comptes par ses services.

Tous les partis y sont donc allés de leurs propositions, en insistant bien sûr, mais du bout des lèvres sur la nécessité de combler le déficit fédéral. On rappellera à cet égard que celui-ci - une paille - s'élève tout de même à plus de 100 milliards de francs. Cette petite pensée faite à l'égard de la dette, quelles sont les propositions faites pour utiliser cet excédent par les quatre partis gouvernementaux?

Pour le PS, un effort doit être fait sur la politique sociale, la santé et l'éducation, en portant l'effort sur le secteur de l'information, mais c'est surtout dans le cadre du financement de l'AVS que le PS souhaite voir se développer les moyens de la Confédération.

En ce qui concerne le PDC, celui-ci réclame la mise en vigueur immédiate de la réforme de la fiscalité des familles.

Le PRD, et cela n'étonnera personne, souhaite s'engager dans une réforme fiscale plus musclée que celle prévue par M. Villiger, et envisage des allége- ments fiscaux dépassant les 1,4 milliards prévus pour atteindre 2 milliards.

Enfin, pour l'UDC, il est nécessaire de prévoir des baisses d'impôts pour tous, l'abolition du droit de timbre et un effort supplémentaire en faveur des familles.

Au sens strict du terme, aucun parti, ni aucun représentant d'aucune formation politique autre que celle représentée au sein du Conseil fédéral n'ont fait de propositions quant à une amélioration $d u$ fonctionnement de l'assurance-maladie et, en particulier aux moyens d'alléger la charge des ménages due au paiement des primes mensuelles.

On se rappellera pourtant que l'augmentation régulière des primes depuis l'introduction de la LAMal, est due non seulement à l'élargissement initial du catalogue des prestations prises en charge dès 1996 ce qui fait du catalogue suisse des prestations le plus élaboré par rapport aux pays qui nous entourent mais également au désengagement massif des pouvoirs publics qui ont cessé de subventionner comme par le passé, notre système social d'assurance-maladie.

Les hausses successives que supportent les ménages à l'heure actuelle sont donc un transfert des charges de la Confédération vers les familles, et il ne serait que justice que les excédents enregistrés dans les comptes 2000 de la Confédération puissent être redistribués afin de soulager ainsi les assurés.

Il ne semble donc que personne n'y ait pensé à l'heure actuelle, sans que l'on sache exactement pourquoi d'ailleurs. Nous avons pourtant esquissé trois réponses possibles, qui sont bien entendu cumulatives:

$\square$ Les assurés ne sont pas représentés dans les quatre partis gouvernementaux.

$\square$ La hausse des primes n'intéresse personne et chacun se contente de la situation existante.

$\square$ Nous ne sommes pas en année électorale.

Cochez ce qui convient et ce sera tout pour aujourd'hui.

Jean-Marc Guinchard Secrétaire général Association des Médecins du canton de Genève 